\title{
When two heads are better than one: Interactive versus independent benefits of collaborative cognition
}

\author{
Allison A. Brennan • James T. Enns
}

Published online: 22 November 2014

(C) Psychonomic Society, Inc. 2014

\begin{abstract}
Previous research has shown that two heads working together can outperform one working alone, but whether such benefits result from social interaction or from the statistical facilitation of independent responses is not clear. Here we apply Miller's (Cognitive Psychology, 14, 247-279, 1982; Ulrich, Miller \& Schröter, Behavior Research Methods, 39(2), 291-302, 2007) race model inequality (RMI) to distinguish between these two possibilities. Pairs of participants completed a visual enumeration task, both as independent individuals and as two members of a team. The results showed that team performance exceeded the efficiency of two individuals working independently, indicating that interpersonal interaction underlies the collaborative gains in this task. This interpretation was bolstered by analyses showing that the magnitude of the collaborative benefit was positively mediated by the strength of social affiliation and by the similarity of verbal communication among team members. This research serves as a proof-of-concept that Miller's RMI can differentiate between interactive versus independent effects of collaborative cognition. Moreover, the finding that social affiliation and communication similarity each contribute to the collaborative benefit suggests new avenues of research for establishing the mechanisms supporting collaborative cognition.
\end{abstract}

Keywords Collaborative cognition · Social coordination . Visual enumeration $\cdot$ Race model inequality $\cdot$ Joint attention . Cooperation

It is well established that two or more individuals can outperform one. For example, the average estimate of the weight of an ox by a crowd is more accurate than the best individual

A. A. Brennan $(\bowtie) \cdot$ J. T. Enns

Department of Psychology, University of British Columbia,

Vancouver, BC, Canada V6T 1Z4

e-mail: abrennan@psych.ubc.ca estimate (Galton, 1907; Surowiecki, 2004). In team problem solving, brainstorming groups generate more solutions than the same number of individuals working independently (Osborn, 1948). In a task with noisy visual signals, pairs who communicate their confidence to one another outperform individuals (Bahrami et al., 2010).

Such findings are interpreted as the benefits of collaborative cognition. However, a closer examination reveals that these benefits can also be obtained from non-communicating team members and without social collaboration. For example, the average of multiple estimates from the same individual can be more accurate than any single best estimate (Herzog \& Hertwig, 2009), and the benefit in noisy signal detection by pairs can be replicated by simply selecting the response of the more confident individual (Koriat, 2012).

These opposing accounts of the benefits of collaborative cognition pose a dilemma. Either the benefits of cognitive teamwork result from social interaction among team members, or they are the expected statistical benefit of aggregating independent responses. To distinguish between these accounts, we applied Miller's (1982; Ulrich, Miller \& Schröter, 2007) race model inequality (RMI). This tool was originally developed to test whether redundant target signals were processed in a single mind as a race between independent activations (with the faster signal determining the response on each occasion) or whether the signals were co-activated (signal activations were combined prior to the response decision).

Several fields have profited from this distinction. For example, research in multisensory integration (Hecht, Reiner, \& Karni, 2008; Murray et al., 2005) has used Miller's RMI to confirm that observers can detect multimodal signals (e.g., combined audio and visual) faster than unimodal signals (e.g., audio or visual alone), because the signals from each modality are integrated prior to the decision to respond. Research on cortical interactions between the hemispheres has used the RMI to confirm the presence of mutual hemispheric inhibition 
(Corballis, 1998; Miller, 2004). This was revealed by paradoxically greater coactivation in redundant signal detection for individuals without a functioning corpus callosum, when compared to coactivation in healthy adults.

Here we use the RMI to determine whether the benefits of collaboration are based merely on statistical facilitation, or whether there is social interaction between team members. To do this, we substituted the comparison of one versus two target-signals in previous studies with a comparison of performance by one versus two people. If performance by twoperson teams does not exceed the model of statistical facilitation derived from treating each individual's performance as an independent signal, the benefit of collaborative cognition merely reflects statistical facilitation. Alternatively, if team performance exceeds the statistical facilitation benchmark, the benefit of collaborative cognition must involve social interaction between individuals. While we test for social collaborative benefits over and above statistical facilitation, previous research has not considered this distinction and thus runs the risk of overestimating the magnitude of collaborative benefits, as illustrated in Table 1 .

We chose a collaborative cognitive task that involved the visual enumeration of a set of targets in a naturalistic setting

Table 1 Three procedures for comparing individual and team performance, using a toy data set of response times (RTs) (msec) on four detection trials

\begin{tabular}{lccccc}
\hline & Trial 1 & Trial 2 & Trial 3 & Trial 4 & Mean \\
\hline Person A = & 200 & 300 & 400 & 200 & 275 \\
Person B = & 300 & 200 & 400 & 500 & 350 \\
Team (Person A + B) $=$ & 225 & 225 & 270 & 240 & 240
\end{tabular}

Brennan et al. (2008) would claim a collaborative benefit because the team mean $(240)$ is faster than the fastest individual on each trial $(250=$ $[200+200+400+200] / 4)$.

Bahrami et al. (2010) claimed a collaborative benefit in accuracy because teams were more accurate than the more accurate individual. Although they did not make specific claims about RTs, we do so here to facilitate comparison of their approach with our procedures. In our understanding, Bahrami et al. would claim a collaborative benefit because the team mean (240) is faster than the faster of the two individual means (275).

Miller's RMI (1982; Ulrich, Miller \& Schröter, 2007) would compare individuals and teams in three steps:

Step 1: Combine RT values from Person A and B [ [llllll $300 \quad 400 \quad 200$ $300 \quad 200 \quad 400 \quad 500]$.

Step 2: Place RT values in ascending order $\left[\begin{array}{lllll}200 & 200 & 200 & 300 & 300\end{array}\right.$ $400400500]$.

Step 3: Find the mean of the first $n$ RT values, where $n=$ the number of trials performed by the team $\left[\begin{array}{llll}200 & 200 & 200 & 300\end{array}\right]=225$.

The team mean (240) is slower than Millers's RMI mean of 225, which is based on a model of statistical facilitation. Therefore, there is no evidence of coactivation in these data, arguing against the team benefits deriving from social interaction. Coactivation is only warranted when the team mean is faster than 225 . for several reasons. First, visual enumeration generates many correct response times (RTs) for each condition, which is required for applying Miller's RMI. Second, this task is limited primarily by speed, not accuracy. Because individuals and pairs can perform the task with equivalently high accuracy, we can explore whether teamwork speeds performance. Third, visual enumeration is sufficiently cognitively complex that it allows for the possibility of collaborative benefits without artificially necessitating social interaction or preventing social interaction.

If we are successful in applying Miller's RMI to the measurement of collaborative cognition, an important next step is to consider the mechanisms that help people work together. Previous research suggests that dividing the cognitive load between collaborators allows a process that is normally executed serially within an individual to be executed more efficiently by a pair working in parallel. Visual enumeration lends itself well to collaboration, considering the capacity limitation of individual attention for visual space and the representation of targets in working memory (Houtkamp \& Roelfsema, 2009). Effective teamwork might therefore divide the task spatially, each member searching roughly half the items (Brennan et al., 2008), or in the case of multiple targets, have each member search for half of the targets (Chen, 2007).

We propose that social factors mediate people's ability to collaborate efficiently. Here we explore two social factors that have been implicated in previous research: the strength of affiliation between pair members and the frequency with which they verbally communicate. Research has shown that familiarity with a collaborator enhanced the establishment of a shared problem-solving space (Barron, 2003) and that verbal communication between team members was required for teams to outperform individuals (Bahrami et al., 2012).

\section{Method}

Participants Forty-four University of British Columbia students (22 pairs; 26 female; mean age 22.6 years) were recruited through advertisements asking for participation in research with a friend in exchange for $\$ 10$. Participants provided written informed consent and were debriefed in accordance with American Psychological Association (APA) guidelines.

Stimuli and apparatus The experimental displays depicted shelves containing 82 distractor objects commonly found in a home or office and 0,1 , or 2 of 4 possible targets (see Fig. 1). Distractors appeared in four different arrangements. The same target never appeared twice in a photograph. Each target appeared equally often in each display quadrant in one target display and in one of the quadrants not already occupied by the first target in two target displays. This generated 356 search displays in total (four displays with no target, 64 with 

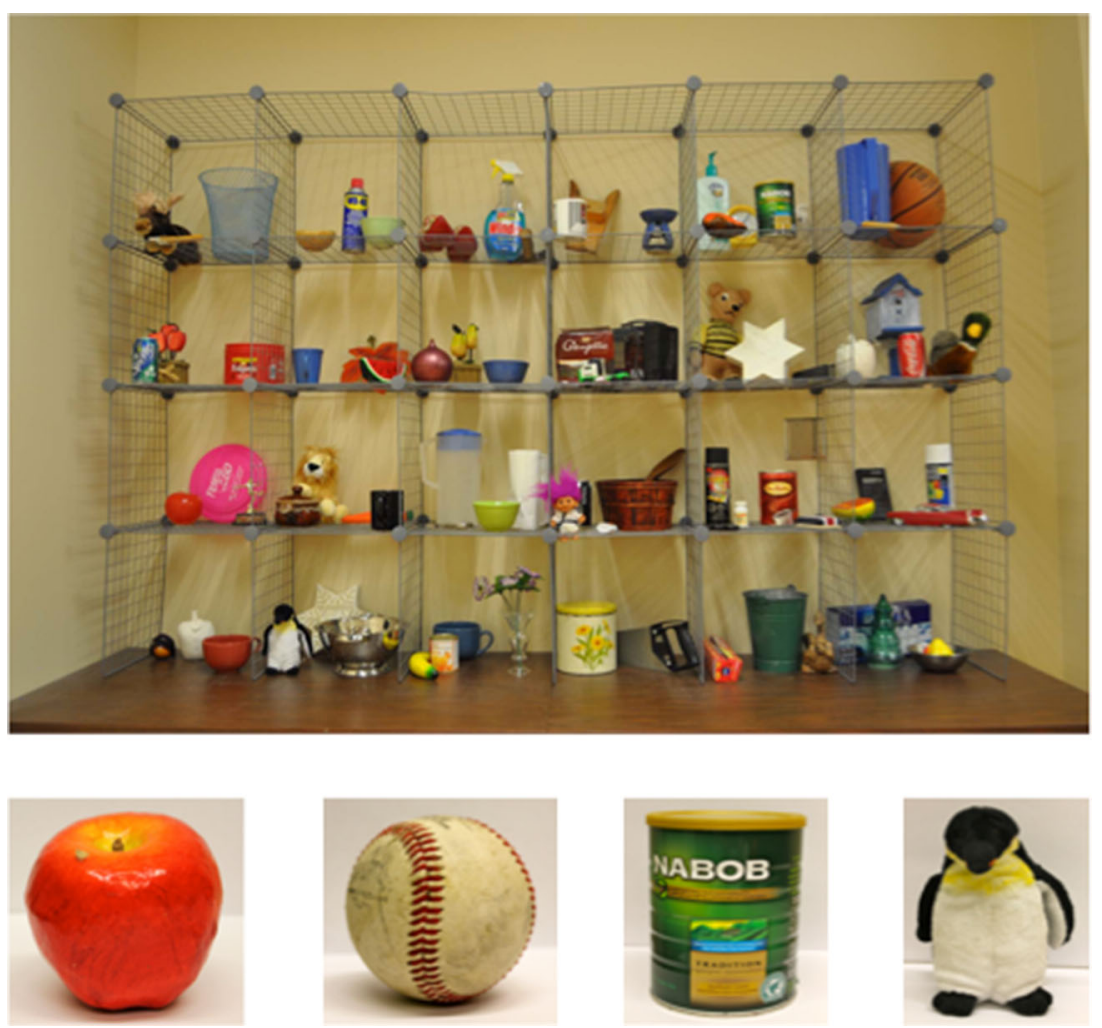

Fig. 1 A typical display in the study (top) and four targets that could appear in each display (bottom). Participants searched displays and indicated whether 0,1 , or 2 targets were present. This display contains 2 targets: coffee can and penguin

one target, and 288 with two targets). Displays subtended a $40^{\circ} \times 32^{\circ}$ visual angle on a 24 -in iMac computer. The experiment was controlled by Matlab 2010a and Psychtoolbox3. An iSight webcam recorded participants' head and torso during the collaborative task.

Procedure Participants indicated as rapidly and accurately as possible the number of targets present. Before testing, they were familiarized with the displays and the four target objects, which remained continuously visible in four pictures placed underneath the computer screen. The reason initially given to participants for video-recording the session was for the purpose of "knowing where your eyes are looking when you search." Upon debriefing, participants were informed that the real reason was "to be able to measure the verbal and nonverbal behavior associated with your performance as a team." Participants were then given the option of declining to have their video data be used in the study, but all agreed.

Half the participants first performed the task individually before being tested as a team; the other half performed as a team before being tested individually. In each case, participants searched a subset of all of possible displays, and indicated the number of targets present by pressing keys labeled 0 , 1 , or 2. Participants completed 90 trials as individuals (30 each with 0,1 , and 2 targets) and 90 trials as a team. We used weighted random sampling of the 356 total search displays to ensure that 0,1 , and 2 target trials appeared with equal frequency. Participants received feedback on their percentage of correct responses every 15 trials.

When searching as a team, participants were instructed to use whatever strategy they thought was best to work together. Because there was only one keyboard for response entry, each participant took a turn entering the response, with the keyboard being exchanged after 45 trials.

Social affiliation measure Following completion of the visual enumeration task, participants completed the Intimate Friendship Scale (IFS; Sharabany, 1974), consisting of 32 questions such as "I can be sure that my friend will help me whenever I ask for it." Responses were made using a 5-point Likert scale. An IFS score for each pair was computed by summing individual participant scores.

Communication similarity measure A research assistant blind to the purpose made a written transcript of all verbal communication during the team task and the total number of distinct utterances made by each team member was tallied. The difference in utterance between team members, divided by the total number of team utterances, was an index of Communication Similarity, with smaller scores indicating greater similarity. 
Data analysis We tested for violations of RMI in the distributions of the correct responses, using the algorithm and MATLAB routines provided in Ulrich et al. (2007). We substituted the cumulative density functions (CDFs) of correct RTs in the two single-signal conditions from Ulrich et al. (2007) with the CDFs of RTs by the faster and slower of the two individuals in each team, respectively. We also substituted the CDF of RT in the redundant signals condition of Ulrich et al. (2007) with the CDF of RTs by each team. To generate the statistical facilitation benchmark, the CDFs of correct RTs by the two independent individuals in each team were combined into a fourth $\mathrm{CDF}$, which was truncated at the number of correct RTs in the two-person team CDF; this represents the fastest performance that could be derived from the two individuals working independently. If two-person teams are significantly faster than the statistical facilitation benchmark, the RMI is violated and compels the interpretation of social interaction. Alternatively, if two-person teams are no faster than this benchmark, the improved performance of two searchers over one is attributed to statistical facilitation. Typically, the RMI is rejected if there is a significant violation at any percentile (Ulrich et al., 2007).

\section{Results}

Collaborative performance exceeded the performance of either individual considered alone Consistent with previous research, two people working together were faster and more accurate than either independent individual. Mean correct RT and proportion correct are shown in Table 2. Correct responses were made in an average of $12.92 \mathrm{sec}(\mathrm{SE}=0.566)$. We used a mixed-design analysis of variance (ANOVA) to test for differences in correct RT, with social condition (slower individual, faster individual, collaborative) and task order (first, second) as between-groups factors and target number $(0,1,2)$ as a repeated measures factor. This revealed that team enumeration was faster than enumeration by either the slower or faster individual $[F(2$, $60)=15.73, p<.01]$. It also showed that enumerating 2 targets was faster than enumerating 1 or 0 targets $[F(2,120)=216.39, p$ $<.01]$. The task done second was also significantly faster than the same task done first, $[F(1,60)=16.39, p<.01]$.

There were two significant interactions. The difference between team and individual RT was greater with 0 and 1 targets than with 2 targets $[F(4,120)=6.51, p<.01]$, and this difference between targets was greater when the tasks were done first than when done second $[F(2,120)=3.41, p<.04]$. No other effects were significant. Most important was the absence of a significant interaction between social condition and task order, or between target number, task order, and social condition [both $\left.F_{\mathrm{S}}<1.0\right]$. This indicates that the task done second was completed approximately $4 \mathrm{~s}$ faster than
Table 2 Mean correct response time (RT) and mean accuracy (SE in parentheses) by the factors in the study: Task Order (first, second), number of targets in the display $(0,1,2)$, and social condition (slower individual, faster individual, collaborative)

\begin{tabular}{|c|c|c|}
\hline \multirow[b]{2}{*}{ Task Order } & \multicolumn{2}{|c|}{ Correct RT (sec) } \\
\hline & $\begin{array}{l}\text { First } \\
0 \text { target }\end{array}$ & Second \\
\hline Slower individual & $20.60(1.54)$ & $17.11(1.72)$ \\
\hline Faster individual & $18.07(1.55)$ & $14.36(1.52)$ \\
\hline Collaborative & $\begin{array}{l}14.08(1.37) \\
1 \text { target }\end{array}$ & $8.88(0.52)$ \\
\hline Slower individual & $20.15(1.55)$ & $16.06(1.59)$ \\
\hline Faster individual & $17.26(1.67)$ & $13.75(1.27)$ \\
\hline Collaborative & $\begin{array}{l}13.35(1.25) \\
2 \text { targets }\end{array}$ & $9.05(0.57)$ \\
\hline Slower individual & $11.45(0.59)$ & $8.72(0.53)$ \\
\hline Faster individual & $8.64(0.72)$ & $7.19(0.32)$ \\
\hline Collaborative & $\begin{array}{l}8.13(0.70) \\
\text { Accuracy (Pro }\end{array}$ & $\begin{array}{l}5.40(0.34) \\
\text { rect })\end{array}$ \\
\hline Task Order & $\begin{array}{l}\text { First } \\
0 \text { target }\end{array}$ & Second \\
\hline Slower individual & 97.64 (1.19) & $99.58(0.32)$ \\
\hline Faster individual & $97.52(0.90)$ & $99.09(0.91)$ \\
\hline Collaborative & $\begin{array}{l}95.82(3.05) \\
1 \text { target }\end{array}$ & $99.70(0.30)$ \\
\hline Slower individual & $92.12(2.30)$ & $93.94(1.55)$ \\
\hline Faster individual & $88.54(2.77)$ & $92.36(3.38)$ \\
\hline Collaborative & $\begin{array}{l}88.18(4.34) \\
2 \text { targets }\end{array}$ & $95.09(2.25)$ \\
\hline Slower individual & $82.24(4.86)$ & $82.85(3.35)$ \\
\hline Faster individual & $74.42(7.50)$ & $82.30(4.81)$ \\
\hline Collaborative & $86.48(2.05)$ & $90.61(2.24)$ \\
\hline
\end{tabular}

when it was done first, but that the size of this benefit did not vary with social condition.

Response accuracy was high at $91.03 \%(\mathrm{SE}=1.22)$. An ANOVA with the same factors as correct RT revealed that accuracy declined as target number increased $[F(2,120)=$ $52.34 p<.01]$. Social condition and target number significantly interacted, such that team accuracy exceeded individual accuracy more with 2 targets than with 1 or 0 targets $[F(4,120)=2.75, p=$ .04]. No other main effects or interactions were significant, although tasks performed second were marginally more accurate than tasks performed first $[F(1,60)=3.27 p<.08]$.

These analyses show that the benefit of teamwork was most evident in RT data for 0 and 1 target and in accuracy data for 2 targets. These data also reveal an overall speedaccuracy tradeoff, such that participants made relatively rapid responses to 2 targets at the expense of accuracy. Because of this, we submitted the high accuracy RTs in the 0- and 1-target conditions to an RMI analysis of correct RT. This included 60 
trials of data for each social condition, minus the small number of trials in which an error occurred.

The benefit of teamwork was the result of social interaction, not statistical facilitation As shown in Fig. 2, team performance surpassed the benchmark of the statistical facilitation model that denoted the fastest possible performance of two independent individuals (see Method: Data analysis). This violation of the RMI indicates that the benefit of collaboration results from social interaction and is not merely the statistical facilitation expected from independent individuals. This conclusion was supported by an overall significant difference between collaborative versus combined CDFs in Fig. $2[\mathrm{~F}(1$, $21)=22.44, p<.01]$, and by Bonferroni-corrected paired sample t-tests at the $10 \mathrm{CDF}$ percentiles. Miller's RMI was violated significantly at percentiles .05 through $.55(t(21)=$ $1.90,2.53,2.43,2.34,2.21,1.92$, respectively, all $p$ 's $<.05)$. Finding that social interaction underlies the benefit of collaborative cognition prefaces the following analysis of the social factors associated with effective team collaboration.

Social affiliation and communication similarity were each associated with collaborative benefits The magnitude of the collaborative benefit for each team was calculated as the mean correct RT of the statistical facilitation model minus the collaborative mean correct RT for each of the ten CDF percentile bins. These values were entered as criterion variables to be regressed against the measures of social affiliation and communication similarity. The regression analyses showed that teams who reported stronger affiliation $(r=.554, p<$ $.01)$ and communicated more equally $(r=.428, p<.01)$ showed larger collaborative benefits at the .05 percentile. As further percentiles were added to the collaborative measure, the strength of these simple correlations declined slightly with each step. However, both correlations remained significant ( $r$ $=.371, p<.05$ and $r=.339, p<.05$, respectively) when all ten percentiles were included. This modest decline is expected since the distinction between the social interaction and statistical facilitation models is greatest for fast RTs in the first CDF percentiles.

A multiple regression analysis predicting the collaborative benefit at the .05 percentile bin showed that social affiliation $(p r=.346, p<.03)$ and communication similarity $(p r=.502, p$ $<.01)$ each contributed independently to the collaborative benefit, with the partial correlation between these predictors near zero $(p r=.048, p>.50)$. Together these predictors explained $39 \%$ of the total variance in the collaborative benefit between teams $(R=.624, \mathrm{~F}(2,39)=12.44, p<.001)$.

\section{Discussion}

This study demonstrates that Miller's (1982; Ulrich et al., 2007) RMI can be used to index collaborative performance benefits that derive from social interaction between people.

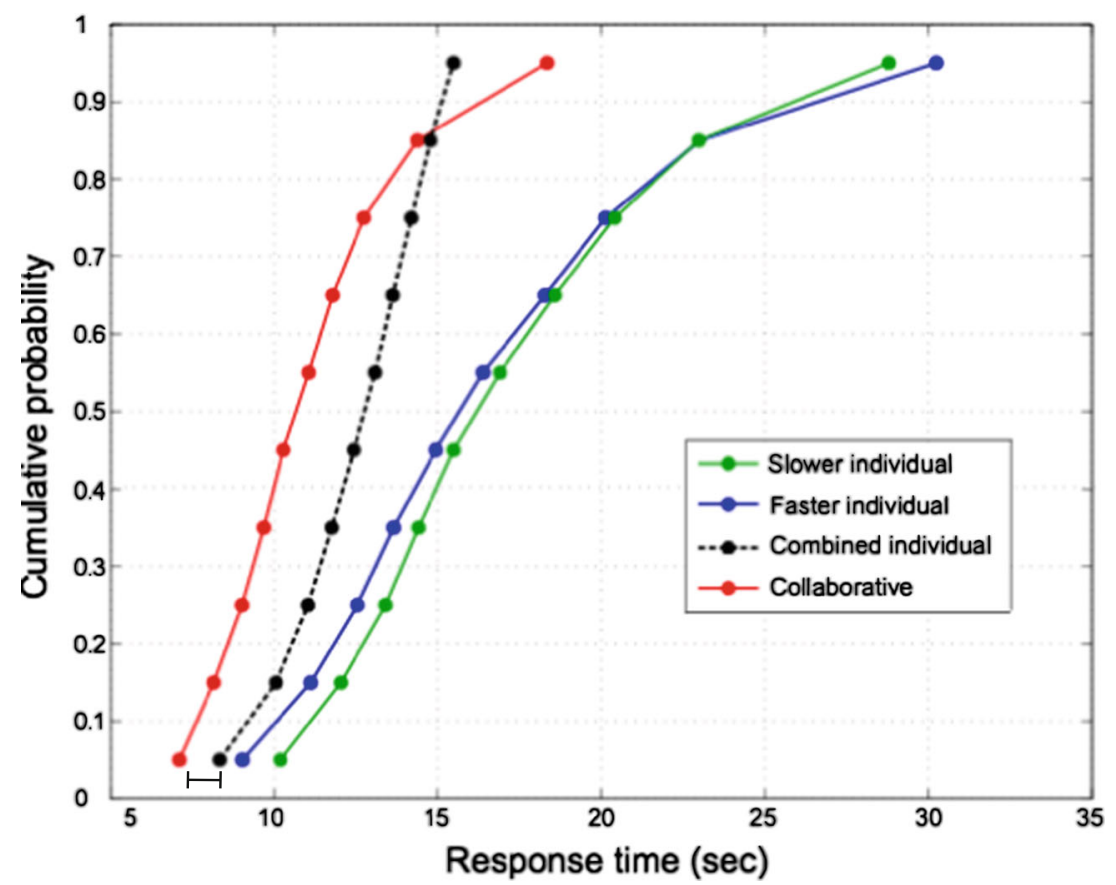

Fig. 2 Mean cumulative density functions for correct response time (RT), averaged over 0 and 1 targets. Faster responses are to the left, slower responses to the right. The speed of two-person teams (red solid line) is not only faster than the faster of two individuals working alone (blue solid line), it exceeds the prediction of statistical facilitation if the same two individuals worked independently (dashed black line). The horizontal bar near the bottom shows the $95 \%$ confidence interval, pooled over all bins, for significant social coactivation 
The RMI was originally developed for the question of how information from redundant target signals is combined within the mind of an individual. As such, it seemed a potentially effective tool for testing whether collaborative performance involves independent or co-activating signals, provided that the signals deriving from different targets in the original model were substituted for signals deriving from different individuals, as in the present study.

When we considered how this approach compared to previous proposals to address this question, it was clear that previous efforts ran the risk of overestimating the magnitude of collaborative performance benefits. This is shown in the example worked out in Table 1, which illustrates how our adaptation of Miller's (1982) RMI framework offers a more rigorous analysis of whether the benefits derive from statistical facilitation or genuine social interaction.

The main result here was that team performance (i.e., two collaborating individuals) exceeded the performance predicted by the same two individuals working independently in a visual enumeration task. This finding implies that social interaction, not statistical facilitation, underlies the benefit of two heads compared to one in this particular task. Although past research has reported that two people outperformed one in other visual search tasks (e.g., Brennan et al., 2008), this is the first rigorous demonstration that the faster responses of teams must be attributed to something more than statistical facilitation.

Two more detailed findings hint at underlying mechanisms of collaborative benefits. First, there was the positive correlation between the strength of social affiliation and the magnitude of the team benefit. Friendship strength in the present study is likely a proxy for deeper mechanisms involved in negotiating joint attention. One such mechanism may be that friendship reduces the overall attentional load incurred when individuals must communicate in a cognitively demanding task. In support of this idea are reports that familiarity with an activity lessens the attentional resources it requires (Beilock, Wierenga, \& Carr, 2002).

The second finding, that communicating at a similar frequency was correlated with larger team benefits, implies that the benefits are greatest when there is a near equitable division of cognitive labor. Our reading of the verbal transcripts indicated that the majority of utterances communicated the identity or location of targets. A similar number of utterances by team members thus implies that they were doing similar amounts of work. It follows that pairs of individuals who undertook similar efforts showed larger collaborative benefits, and that teams with unequal efforts were hampered by the increased cognitive expenditure and communication requirements for one of the individuals. This is consistent with Bahrami et al.'s (2010) report that teams comprised of individuals with more similar visual sensitivities collaborated more effectively than partners with disparate visual sensitivities. However, unlike the present study where team members viewed the same display and varied from one another naturally, Bahrami et al. (2010) artificially reduced the visual contrast of the display seen by one partner in order to directly manipulate this factor.

Limitations and future directions The present findings come from a visual enumeration task that generates many correct RT data points and few errors. This produces the required inputs for testing Miller's RMI, but at the same time, it raises the question of how generally the conclusions can be applied. An important step in future research will be to extend the statistical logic of the RMI to tasks that measure accuracy or perceptual sensitivity rather than RT. Fortunately, research on perceptual decision-making in individuals has already laid that groundwork. For example, Schwarz and Miller (2014) discuss the interactive versus independent effects of redundant signals on response accuracy, recommending that both hits and false alarms be considered with equal rigor in any analysis. In the realm of perceptual sensitivity at threshold, researchers have applied the statistical logic of Miller's RMI to study the perception of facial expressions based on single features and combinations of features (Gold, 2014). These measures also seem ripe for use by researchers of collaborative cognition.

Although the RMI provides an appropriate rigorous baseline for comparing individual and team performance, we caution that it may be conservative because social collaboration has both benefits and costs. On the one hand, cognition can be made more efficient when collaborators divide the cognitive load (Houtkamp \& Roelfsema, 2009). On the other hand, the coordination of cognition through speech or gesture also incurs a cost (Brennan et al., 2008). It is therefore likely that some mix of benefits and costs is captured in the present measure of collaboration. As such, team performance might fail to exceed the statistical facilitation model simply because the combined cost of collaboration outweighs the benefit, not because social interaction was absent. Teasing apart these factors remains an important question for future research.

Because this study measures the magnitude of collaborative benefits during a timed visual enumeration task, it is difficult to directly compare the magnitude of the gains reported here to those found in previous research. The many differences remaining to be explored include: (1) the measure of performance (e.g., RT, accuracy, sensitivity), (2) the benchmark used for assessing team performance (e.g., statistical facilitation, better of two individuals, nominal pairs), (3) the nature of the task (e.g., visual enumeration, contrast sensitivity, search), and (4) the constraints imposed on collaboration (e.g., unstructured, highly structured).

The present demonstration that social affiliation and communication similarity are each associated with benefits in a collaborative cognition task is an important first step in understanding how social interaction benefits cognition. We 
readily acknowledge that other aspects of social interaction may be involved, especially as collaborative cognition is studied in a wider range of settings and tasks. For example, it is possible that affiliated pairs were more aligned in their vocabulary (Fusaroli et al., 2012), and/or in their body movement (e.g., posture, gesture, eye movement; Lumsden, Miles, Richardson, Smith, \& Macrae, 2012), and that alignment on these dimensions facilitated collaborative performance gains. We hope that the groundwork for these questions has now been laid.

Author Note This research was supported by grants from the Natural Sciences and Engineering Research Council of Canada to J.T. Enns (Discovery Grant) and A.A. Brennan (PhD Training). We thank Chris Yeh, Ema Salja, Veronica Galvin, Sarah MacDonald, and Craig Zhou for testing participants and video transcription, and Peter Lenkic, Ana Pesquita, and Marcus Watson for programming and comments.

\section{References}

Bahrami, B., Olsen, K., Latham, P. E., Roepstorff, A., Rees, G., \& Frith, C. D. (2010). Optimally interacting minds. Science, 329, 10811085.

Bahrami, B., Olsen, K., Bang, D., Roepstorff, A., Rees, G., \& Frith, C. D. (2012). Together, slowly, but surely: The role of social interaction and feedback on the build-up of benefit in collective decisionmaking. Journal of Experimental Psychology: Human Perception and Performance, 38(1), 3-8.

Barron, B. (2003). When smart groups fail. Journal of Learning Sciences, 12(3), 307-359.

Beilock, S. L., Wierenga, S. A., \& Carr, T. H. (2002). Expertise, attention, and memory in sensorimotor skill execution: Impact of novel task constraints on dual-task performance and episodic memory. Quarterly Journal of Experimental Psychology Section A Human Experimental Psychology, 55(4), 1211-1240.

Brennan, S. E., Chen, X., Dickinson, C. A., Neider, M. B., \& Zelinsky, G. J. (2008). Coordinating cognition: The costs and benefits of shared gaze during collaborative search. Cognition, 106, 1465-1477.

Chen, X. (2007). Collaborative visual search. (Doctoral dissertation). Retrieved from SUNY Digital Repository. (Accession Order No. 1951/44780)
Corballis, M. C. (1998). Interhemispheric neural summation in the absence of the corpus callosum. Brain, 121, 1795-1807.

Fusaroli, R., Bahrami, B., Olsen, K., Roepstorff, A., Rees, G., Frith, C., \& Tylen, K. (2012). Coming to terms: Quantifying the benefits of linguistic coordination. Psychological Science, 23(8), 931-939.

Galton, F. (1907). Vox populi. Nature, 75, 450-451.

Gold, J. M. (2014). Perceptually completed whole is less than the sum of its parts. Psychological Science, 25(6), 1206-1217.

Hecht, D., Reiner, M., \& Karni, A. (2008). Enhancement of response time to bi- and tri-modal sensory stimuli during active movements. Experimental Brain Research, 185(4), 655-665.

Herzog, S. M., \& Hertwig, R. (2009). The wisdom of many in one mind: Improving individual judgments with dialectical bootstrapping. Psychological Science, 20(2), 231-237.

Houtkamp, R., \& Roelfsema, P. R. (2009). Matching of visual input to only one item at any one time. Psychological Research, 73, 317326.

Koriat, A. (2012). When are two heads better than one and why? Science, $336,360$.

Lumsden, J., Miles, L. K., Richardson, M. J., Smith, C. A., \& Macrae, C. N. (2012). Who syncs? Social motives and interpersonal coordination. Journal of Experimental Social Psychology, 48, 746-751.

Miller, J. (1982). Divided attention: Evidence for coactivation with redundant signals. Cognitive Psychology, 14, 247-279.

Miller, J. (2004). Exaggerated redundancy gain in the split brain: A hemispheric coactivation account. Cognitive Psychology, 49, 118154.

Murray, M. M., Molholm, S., Michel, C. M., Heslenfeld, D. J., Ritter, W., Javitt, D. C., ... Foxe, J. J. (2005). Grabbing your ear: Rapid auditory-somatosensory interactions in low-level sensory cortices are not constrained by stimulus alignment. Cerebral Cortex, 15(7), 963-974.

Osborn, A. F. (1948). Your Creative Power. New York: Scribner.

Schwarz, W., \& Miller, J. O. (2014). When less equals more: Probability summation without sensitivity improvement. Journal of Experimental Psychology: Human Perception and Performance, 40(5), 2091-2100.

Sharabany, R. (1974). Intimate Friendship among Kibbutz and City Children and its Measurement. PhD dissertation, Cornell University, No. 74-17-682, Ann Arbor (MI 48106): University Microfilms International.

Surowiecki, J. (2004). The wisdom of crowds. New York: Random House.

Ulrich, R., Miller, J., \& Schröter, H. (2007). Testing the race model inequality: An algorithm and computer programs. Behavior Research Methods, 39(2), 291-302. 